\title{
Coupling of flexural and longitudinal wave motion in a periodic structure with asymmetrically arranged transverse beams
}

Friis, Lars; Ohlrich, Mogens

Published in:

Acoustical Society of America. Journal

Link to article, DOI:

$10.1121 / 1.2065767$

Publication date:

2005

Document Version

Publisher's PDF, also known as Version of record

Link back to DTU Orbit

Citation (APA):

Friis, L., \& Ohlrich, M. (2005). Coupling of flexural and longitudinal wave motion in a periodic structure with asymmetrically arranged transverse beams. Acoustical Society of America. Journal, 118(5), 3010-3020. https://doi.org/10.1121/1.2065767

\section{General rights}

Copyright and moral rights for the publications made accessible in the public portal are retained by the authors and/or other copyright owners and it is a condition of accessing publications that users recognise and abide by the legal requirements associated with these rights.

- Users may download and print one copy of any publication from the public portal for the purpose of private study or research.

- You may not further distribute the material or use it for any profit-making activity or commercial gain

- You may freely distribute the URL identifying the publication in the public portal 


\title{
Coupling of flexural and longitudinal wave motion in a periodic structure with asymmetrically arranged transverse beams
}

\author{
Lars Friis $^{\text {a) }}$ and Mogens Ohlrich ${ }^{\text {b) }}$ \\ Acoustic Technology, Ørsted $\bullet$ DTU, Technical University of Denmark, Building 352, \\ DK-2800 Kgs. Lyngby, Denmark
}

(Received 1 December 2004; revised 15 August 2005; accepted 22 August 2005)

\begin{abstract}
In this paper we investigate the coupling of flexural and longitudinal wave motions in a waveguide with structural side branches attached at regular intervals. The analysis is based on periodic structure theory, and considers wave transmission in a fully tricoupled and semidefinite periodic assembly of beam-type elements (or plane-wave transmission for normal incidence in a similar plate assembly). Receptances of a composite periodic element with offset resonant beams are derived and used for computing the frequency-dependent propagation constants of three coupled wave types as well as the distribution of motion displacements in each wave type. This is used for calculating the spatial variation of the forced harmonic responses of a semi-infinite periodic structure to point excitations by a longitudinal force and by a moment. Numerical simulations reveal the complicated wave coupling phenomena, which are clarified by calculating the ratio of flexural and longitudinal kinetic energies in the wave-carrying component for each wave type. In contrast to a corresponding, but uncoupled, system with significant broadband attenuation of flexural waves, the numerical results further show that the flexural-longitudinal wave coupling in a system with resonant side branches results in a highly enhanced wave transmission with very little attenuation from element to element. (C) 2005 Acoustical Society of America. [DOI: 10.1121/1.2065767]
\end{abstract}

PACS number(s): 43.40.At, 43.40.Cw, 43.20.Bi [MO]

Pages: $3010-3020$

\section{INTRODUCTION}

Many types of engineering structures are built up of an assembly of nominally identical elements that are coupled together in an identical manner to form a so-called "spatially periodic structure." Examples are offshore accommodation modules, ship structures, and some buildings. The vibration and transmission of structural waves, say, from floor to floor in multistory buildings or from deck to deck in a ship, often give rise to noise problems in removed areas. A full threedimensional analysis of such audiofrequency problems is very difficult due to the vibrational interactions between the large numbers of structural components. However, a fundamental understanding of the transmission may be obtained from studies of less complicated models that consider only a single transmission path comprising an assembly of beamtype components, or assume plane-wave transmission for normal incidence in a similar periodic assembly of plate elements.

A periodic system composed of repeated elements that are coupled with one another through $n$ motion coordinates is known to support $n$ characteristic-free, harmonic wave-types, which can exist simultaneously and independently at any frequency. ${ }^{1-3}$ These wave-types occur in $n$ pairs of positiveand negative-going waves, where each of the waves types is governed by a pair of complex characteristic "propagation constants" $\mu= \pm\left(\mu_{R}+i \mu_{I}\right)$; the real part $\mu_{R}$ is the so-called attenuation constant and the imaginary part $\mu_{I}$ is the phase constant. Here $\mu_{R}$ and $\mu_{I}$ are defined as positive. So, if only

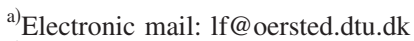

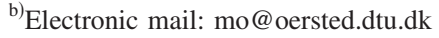

a single positive-going characteristic harmonic wave with propagation constant $\mu=-\left(\mu_{R}+i \mu_{I}\right)$ and angular frequency $\omega$ travels through the system, then the complex displacements $q(x)=q(\xi)$ and $q(\xi+l)$ at identical positions $\xi$ in adjacent elements of length $l$ are related by $q(\xi+l) e^{i \omega t}=e^{\mu} q(\xi) e^{i \omega t}$. This shows that free wave motion is possible only in frequency bands where $\mu$ is purely imaginary. These bands are known as "propagation zones" or "pass bands." For negligible structural damping, the wave thus propagates throughout the system without a change in amplitude. The frequency bands in which $\mu$ is real are called "attenuation zones" or "stop bands," since no transport of vibrational energy is possible and the wave amplitude is attenuated (reduced) from element to element.

Some years ago Ohlrich ${ }^{4}$ investigated the propagation characteristics of longitudinal and flexural waves in beam- or column-type transmission paths with symmetrically arranged "point loads" in the form of resonant transverse beams. With such symmetrical cross beams the longitudinal wave motion in the structure was effectively uncoupled from flexural wave motion. This structure was used as a model of an inner or center-core-type transmission path in multistory buildings with floor supporting beams. For typical dimensions it was found that longitudinal waves propagate at almost all frequencies in the low-frequency range, say up to $300 \mathrm{~Hz}$, only being interrupted by narrow-band, resonant attenuation zones caused by modes of the cross beams. Flexural waves were found to be more effectively attenuated by both resonant and smooth attenuation zones, and wave propagation occurred only in narrow bands constituting about $20 \%$ of the considered frequency range. A brief description of these findings is given in Ref. 5. However, experimental results also revealed ${ }^{4}$ 
that inertia loads attached on one side only of the transverse beams provoked longitudinal-flexural wave coupling because of the introduced eccentricity or asymmetry. The implication of this was a significant enhancement of the overall transmission of locally excited flexural wave motion, due to the conversion of flexural wave motion into predominantly longitudinal wave motion, which is generally transmitted with much less attenuation.

An understanding of the wave coupling that occurs in asymmetrically loaded transmission paths is of considerable practical interest, for example, in the prediction of vibration levels and structure-borne sound transmission in webstiffened panels, in ship hulls that have deck structures to one side only and in outer supporting column structures in building skeletons or façade panels of buildings. In such periodic structures the longitudinal and flexural waves will, in general, be fully coupled, which significantly complicates the associated propagation properties and the underlying analysis, as will be revealed in this paper.

Wave motion in various kinds of periodic structures such as multisupported beams, plates, and shells have been studied for many years, but apparently no work has been done on the addressed problem of longitudinal-flexural wave coupling in semidefinite periodic structures with asymmetrical and multiresonant structural components, as are found in practical structures with resonating cross members such as one-sided decks, deep ribs, webs, and the like. Nearly half a century ago, Müller ${ }^{6}$ observed an enhanced wave transmission in rib-reinforced concrete floors and this behavior was modeled by using eccentrically attached inertia loads at regular intervals on an otherwise continuous beam. In addition to Ohlrich's experimental observation, ${ }^{4}$ Mead and Markus ${ }^{7}$ later demonstrated the occurrence of longitudinal-flexural wave conversion in a simply multisupported beam loaded eccentrically with simple oscillators on levers. Their study also revealed that wave coupling diminishes as the structural damping is increased. Coupling of different wave types in periodic structures with lumped point loads has been studied in Refs. 8-11. Manfred Heckl ${ }^{8}$ demonstrated four methods for calculating the structure-borne sound propagation in beams with many nonresonant discontinuities. In three of the methods coupling between longitudinal and flexural waves was taken into account. More recently, Maria Heckl ${ }^{9}$ modeled a profiled cladding as a periodic structure consisting of long narrow panels attached to each other. Propagation characteristics of the coupled flexural-longitudinal wave motion and the transmission of airborne sound through the cladding were calculated numerically. Maria Heckl ${ }^{10}$ also presented a mathematical model for the propagation and coupling of waves in a periodically supported Timoshenko beam. This coupling included flexural, longitudinal, and torsional waves. Roy and Plunkett ${ }^{11}$ used transfer matrices to examine attenuation of flexural waves in an undamped beam with flexible but nonresonant ribs, which were attached either symmetrically or asymmetrically. However, they did not consider coupling between flexural and longitudinal waves.

In the present paper we examine such longitudinalflexural wave coupling in periodic structures with asymmetrical and resonant point loadings caused by continuous

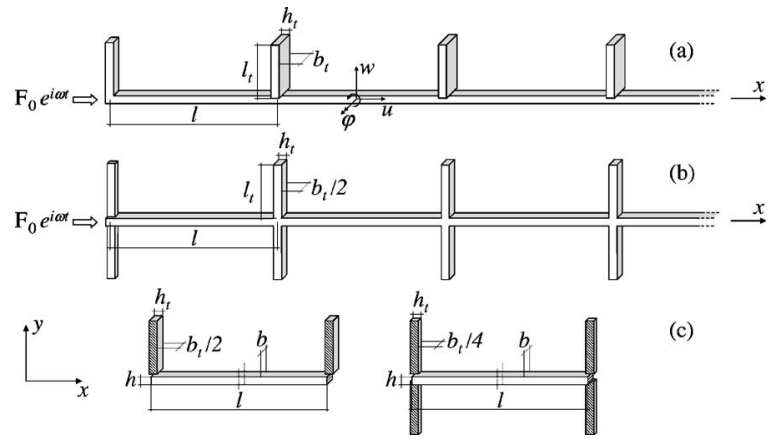

FIG. 1. Semi-infinite periodic structures with load components in the form of (a) asymmetrical beam loadings and (b) symmetrical beam loadings. (c) Corresponding periodic elements that are symmetrical with respect to $x$ $=l / 2$.

cross-members. An investigation of this important problem has not been done before to the best of our knowledge. The effect of coupling on wave propagation and response levels in this type of semidefinite system is investigated and the results obtained are compared with the properties of a similar, but symmetrically loaded, periodic structure in order to ease the understanding of the wave conversion. Both types of structures are presented in Sec. II. The employed analytical analysis is based on the receptance approach to the periodic structure theory developed by Mead. ${ }^{3,12,13}$ This theory is briefly summarized in the beginning of Sec. III for ease of reference, and as a basis for the analysis that follows. Basically, this approach utilizes that harmonic displacements and forces of a single periodic element are related by its dynamic receptances. Therefore, in Sec. III we derive expressions for the receptances of a composite periodic element that are required for determining the propagation constants of the considered type of discrete periodic structure. Next, expressions are presented for determining the coupled response of semiinfinite periodic systems from the eigenvectors of the characteristic wave types. This technique, which was developed by Mead ${ }^{13}$ utilizes so-called normalized force vectors and displacement vectors together with a set of generalized wave coordinates. This is put into use in Sec. IV, where receptances, propagation constants, and total response are derived for the target structure, the asymmetrically point-loaded periodic structure. Finally, the results of a numerical investigation are presented in Sec. V for a choice of structural parameters that clearly illustrates the longitudinal-flexural wave coupling. In a companion paper ${ }^{14}$ the response of a similar, but finite periodic structure will be examined and predicted responses will be compared with experimental results.

\section{STRUCTURES WITH PERIODIC CROSS-MEMBERS}

Consider a semi-infinite periodic structure that extends to infinity in the positive $x$ direction, as shown in Fig. 1(a). The structure is driven at the end by an external forcing vector $\mathbf{F}_{0} e^{i \omega t}$ that represents both longitudinal and transverse force excitations as well as moment excitation. The response is governed by three motion degrees of freedom, comprising displacements $u(x, t), w(x, t)$, and $\varphi(x, t)$ in the longitudinal, transverse, and rotational directions. Note that the application of any single one of the external force components will 


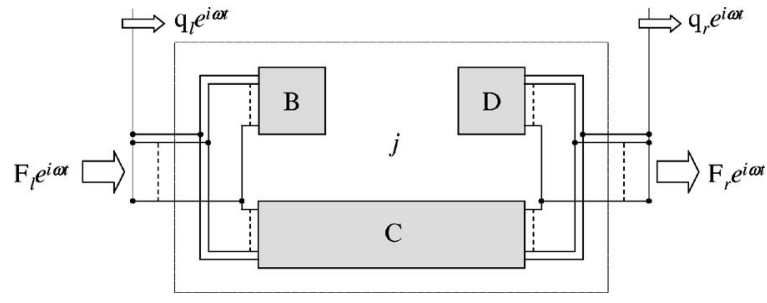

FIG. 2. Free body diagram of a multicoupled, composite periodic element, consisting of a wave-carrying component $\mathrm{C}$ and load components $\mathrm{B}$ and $\mathrm{D}$. Shown are the force vectors on a single periodic element and the associated displacement vectors.

generate a mixed response comprising both longitudinal and flexural wave motions in the column components of such an asymmetric periodic structure. In order to comprehend the effects of coupling between flexural and longitudinal wave motions, it is useful to compare the derived results of response and propagation characteristics with those of a periodic structure in which longitudinal and flexural wave motions are not coupled. Figure 1(b) shows such a structure consisting of a continuous column loaded periodically with symmetrically arranged transverse beams. This type of structure is similar to one previously investigated in Refs. 4 and 5. Due to the symmetrical loadings, longitudinal and flexural wave motions are uncoupled, and excitation by either an external longitudinal (axial) force or a moment thus solely generates longitudinal wave motions or flexural wave motions, respectively. The periodically attached transverse beams have the same weight and same natural frequencies of antisymmetric and pinned modes as those of the structure in Fig. 1(a).

The periodic elements of the two structures are shown in Fig 1(c); for convenience in analysis these elements are chosen to be symmetrical about the middle of the column component, which means that the periodic element can be rotated about the $y$ axis without changing its dynamic properties. This is achieved by dividing each transverse beam into beams of half-width. Thus, when periodic elements are physically connected to one another, the transverse beams of half-width become interconnected to form transverse beams of full width.

\section{THEORY}

\section{A. Outline of theory}

In a general one-dimensional periodic system, each periodic element is coupled at either end to the adjacent element through $n$ motion coordinates. These $n$ coordinates at the left- and right-hand end of the element are represented by the generalized displacement vectors $\mathbf{q}_{l}$ and $\mathbf{q}_{r}$, each being a column vector with $n$ entries. Forces acting on the element's ends are similarly denoted by the force vectors $\mathbf{F}_{l}$ and $\mathbf{F}_{r}$, as illustrated in Fig. 2. This shows a free body diagram of a periodic element consisting of three components, being the wave-carrying component $C$ and two structural loadings, components $B$ and $D$. By assuming harmonic wave motion at angular frequency $\omega$, we can relate the complex displacements $\mathbf{q}$ and forces $\mathbf{F}$ through a $2 n \times 2 n$ receptance matrix $\boldsymbol{\alpha}$ of the periodic element as ${ }^{15} \mathbf{q} e^{i \omega t}=\boldsymbol{\alpha} \mathbf{F} e^{i \omega t}$. With the time de- pendence suppressed this gives, in a partitioned form,

$$
\left(\begin{array}{c}
\mathbf{q}_{l} \\
\mathbf{q}_{r}
\end{array}\right)=\left[\begin{array}{ll}
\boldsymbol{\alpha}_{l l} & \boldsymbol{\alpha}_{l r} \\
\boldsymbol{\alpha}_{r l} & \boldsymbol{\alpha}_{r r}
\end{array}\right]\left(\begin{array}{l}
\mathbf{F}_{l} \\
\mathbf{F}_{r}
\end{array}\right),
$$

where $\boldsymbol{\alpha}_{l l}$ and $\boldsymbol{\alpha}_{r r}$ are the direct receptance matrices of the periodic element, and $\boldsymbol{\alpha}_{l r}$ and $\boldsymbol{\alpha}_{r l}$ are the corresponding transfer receptance matrices. The first subscript refers to the response location and the second to the point of applied unit force excitation (other forces are absent). For linear elastic systems we have $\boldsymbol{\alpha}_{l r}=\boldsymbol{\alpha}_{r l}^{T}$, where superscript $T$ denotes a "transposed" matrix.

If only a single characteristic wave travels through a periodic structure, then continuity of displacements and equilibrium of forces at the ends of a periodic element ensure that the displacement vectors $\mathbf{q}_{l}$ and $\mathbf{q}_{r}$, and force vectors $\mathbf{F}_{l}$ and $\mathbf{F}_{r}$, are related by ${ }^{3}$

$$
\mathbf{q}_{r}=e^{\mu} \mathbf{q}_{l}, \quad \mathbf{F}_{r}=-e^{\mu} \mathbf{F}_{l},
$$

where $\mu=\mu_{R}+i \mu_{I}$ is the complex, frequency-dependent propagation constant. The real part of $\mu$, the "attenuation constant" $\mu_{R}$, expresses the decay rate in wave amplitude per element, whereas the imaginary part, the "phase constant" $\mu_{I}$, describes the phase change of the wave motion per element. Equations (1) and (2) yield a relationship between displacements and forces at the same position, i.e.,

$$
\mathbf{q}_{l}=\left[\boldsymbol{\alpha}_{l l}-e^{\mu} \boldsymbol{\alpha}_{l r}\right] \mathbf{F}_{l} .
$$

From the same equations a general system equation can be derived, giving

$$
\left[\boldsymbol{\alpha}_{l l}+\boldsymbol{\alpha}_{r r}-e^{\mu} \boldsymbol{\alpha}_{l r}-e^{-\mu} \boldsymbol{\alpha}_{r l}\right] \mathbf{F}_{l}=\mathbf{0} .
$$

This represents a quadratic eigenvalue problem for $e^{\mu}$; for nontrivial solutions the determinant of this matrix must be zero,

$$
\left|\boldsymbol{\alpha}_{l l}+\boldsymbol{\alpha}_{r r}-e^{\mu} \boldsymbol{\alpha}_{l r}-e^{-\mu} \boldsymbol{\alpha}_{r l}\right|=0 .
$$

At any given frequency this yields up to $n$ different pairs of propagation constants $\mu= \pm \mu_{i}$, where $i=1,2, \ldots, n$. If the real and imaginary parts of $\mu_{i}$ are defined as positive, then the corresponding $n$ positive-going waves have propagation constants $\mu_{i,+}=-\mu_{i}$, and the $n$ negative-going waves are associated with the propagation constants $\mu_{i,-}=\mu_{i}$.

\section{B. Receptances of a composite periodic element}

Receptances of a periodic element, expressed explicitly in terms of the component receptances, can be derived from the free body diagram in Fig. 2. Displacements and internal forces at the coupling points of the wave-carrying component $C$ are in a similar manner to Eq. (1) related through the $2 n \times 2 n$ receptance matrix $\gamma$ as

$$
\left(\begin{array}{c}
\mathbf{q}_{l, C} \\
\mathbf{q}_{r, C}
\end{array}\right)=\left[\begin{array}{ll}
\boldsymbol{\gamma}_{l l} & \boldsymbol{\gamma}_{l r} \\
\boldsymbol{\gamma}_{r l} & \boldsymbol{\gamma}_{r r}
\end{array}\right]\left(\begin{array}{c}
\mathbf{F}_{l, C} \\
\mathbf{F}_{r, C}
\end{array}\right) .
$$

Further, the displacements and forces of the load components $B$ and $D$ are related by the $n \times n$ receptance matrices $\boldsymbol{\beta}$ and $\boldsymbol{\delta}$ as

$$
\mathbf{q}_{B}=\boldsymbol{\beta} \mathbf{F}_{B},
$$




$$
\mathbf{q}_{D}=\boldsymbol{\delta} \mathbf{F}_{D}
$$

Combining Eqs. (6)-(8) and including the continuity of displacements and equilibrium of forces, the receptance submatrices of the periodic element yield ${ }^{4}$

$$
\begin{aligned}
& \boldsymbol{\alpha}_{r l}=\mathbf{D}^{-1}, \\
& \boldsymbol{\alpha}_{l l}=\left[-\boldsymbol{\gamma}_{l r} \boldsymbol{\delta}^{-1}+\gamma_{l l} \boldsymbol{\gamma}_{r l}^{-1}\left[\mathbf{I}+\boldsymbol{\gamma}_{r r} \boldsymbol{\delta}^{-1}\right]\right] \mathbf{D}^{-1}, \\
& \boldsymbol{\alpha}_{l r}=\left(\mathbf{D}^{-1}\right)^{T}, \\
& \boldsymbol{\alpha}_{r r}=\left[-\boldsymbol{\gamma}_{r l} \boldsymbol{\beta}^{-1}+\gamma_{r r} \boldsymbol{\gamma}_{l r}^{-1}\left[\mathbf{I}+\boldsymbol{\gamma}_{l l} \boldsymbol{\beta}^{-1}\right]\right]\left(\mathbf{D}^{-1}\right)^{T},
\end{aligned}
$$

where

$$
\mathbf{D}=-\boldsymbol{\beta}^{-1} \boldsymbol{\gamma}_{l r} \boldsymbol{\delta}^{-1}+\left[\boldsymbol{\beta}^{-1} \boldsymbol{\gamma}_{l l}+\mathbf{I}\right] \boldsymbol{\gamma}_{r l}^{-1}\left[\mathbf{I}+\boldsymbol{\gamma}_{r r} \boldsymbol{\delta}^{-1}\right]
$$

Here superscript -1 denotes a matrix inversion and $\mathbf{I}$ is the unity matrix.

Submatrices of symmetric periodic elements take on special forms, ${ }^{13}$ and it is thus mathematically convenient to consider the load components as being identical, i.e., $\boldsymbol{\beta}=\boldsymbol{\delta}$, and to separate the end displacements into two types of motion coordinates that are denoted as type I and type II. In a symmetrical flexural mode, for example, the transverse displacements at the ends have the same sign whereas rotations have opposite signs. All kinds of motion coordinates belong to one of these categories, which are denoted type I and type II coordinates, respectively. It is readily seen that longitudinal displacements are type II coordinates. By using this, the displacements and forces in Eqs. (1)-(4) can be written in the following partitioned form:

$$
\mathbf{q}_{l}=\left(\begin{array}{c}
\mathbf{q}_{l_{\mathrm{I}}} \\
\mathbf{q}_{l_{\mathrm{II}}}
\end{array}\right), \quad \mathbf{q}_{r}=\left(\begin{array}{c}
\mathbf{q}_{r_{\mathrm{I}}} \\
\mathbf{q}_{r_{\mathrm{II}}}
\end{array}\right), \quad \mathbf{F}_{l}=\left(\begin{array}{c}
\mathbf{F}_{l_{\mathrm{I}}} \\
\mathbf{F}_{l_{\mathrm{II}}}
\end{array}\right), \quad \mathbf{F}_{r}=\left(\begin{array}{c}
\mathbf{F}_{r_{\mathrm{I}}} \\
\mathbf{F}_{r_{\mathrm{II}}}
\end{array}\right) .
$$

Likewise, the periodic element's subreceptance matrices can be partitioned with respect to type I and II coordinates and the following relationships can be shown to apply: ${ }^{13}$

$$
\begin{aligned}
& \boldsymbol{\alpha}_{l l}=\left[\begin{array}{cc}
\boldsymbol{\alpha}_{l l_{\mathrm{I}, \mathrm{I}}} & \boldsymbol{\alpha}_{l l_{\mathrm{I}, \mathrm{II}}} \\
\boldsymbol{\alpha}_{l l_{\mathrm{I}, \mathrm{II}}}^{T} & \boldsymbol{\alpha}_{l l_{\mathrm{II}, \mathrm{II}}}
\end{array}\right], \\
& \boldsymbol{\alpha}_{r r}=\left[\begin{array}{cc}
\boldsymbol{\alpha}_{l l_{\mathrm{I}, \mathrm{I}}} & -\boldsymbol{\alpha}_{l l_{\mathrm{I}, \mathrm{II}}} \\
-\boldsymbol{\alpha}_{l l_{\mathrm{I}, \mathrm{II}}}^{T} & \boldsymbol{\alpha}_{l l_{\mathrm{II}, \mathrm{II}}}
\end{array}\right], \\
& \boldsymbol{\alpha}_{l r}=\boldsymbol{\alpha}_{r l}^{T}=\left[\begin{array}{cc}
\boldsymbol{\alpha}_{l r_{\mathrm{I}, \mathrm{I}}} & \boldsymbol{\alpha}_{l r_{\mathrm{I}, \mathrm{II}}} \\
-\boldsymbol{\alpha}_{l r_{\mathrm{I}, \mathrm{II}}^{T}}^{T} & \boldsymbol{\alpha}_{l r_{\mathrm{II}, \mathrm{II}}}
\end{array}\right] .
\end{aligned}
$$

Herein submatrices $\boldsymbol{\alpha}_{l l_{\mathrm{I}, \mathrm{I}}}, \boldsymbol{\alpha}_{l l_{\mathrm{II}, \mathrm{II}}}, \boldsymbol{\alpha}_{l r_{\mathrm{I}, \mathrm{I}}}$, and $\boldsymbol{\alpha}_{l_{\mathrm{II}, \mathrm{II}}}$ are all symmetric. Thus, in the case of systems with symmetric periodic elements the receptance submatrices given by Eq. (15) lead to the following special forms of equations, Eqs. (4) and (5):

$$
\begin{aligned}
& {\left[\begin{array}{cc}
\boldsymbol{\alpha}_{l l_{\mathrm{I}, \mathrm{I}}} & \mathbf{0} \\
\mathbf{0} & \boldsymbol{\alpha}_{l l_{\mathrm{II}, \mathrm{II}}}
\end{array}\right]-\cosh \mu\left[\begin{array}{cc}
\boldsymbol{\alpha}_{l r_{\mathrm{I}, \mathrm{I}}} & \mathbf{0} \\
\mathbf{0} & \boldsymbol{\alpha}_{l r_{\mathrm{II}, \mathrm{II}}}
\end{array}\right]} \\
& \left.-\sinh \mu\left[\begin{array}{cc}
\mathbf{0} & \boldsymbol{\alpha}_{l r_{\mathrm{I}, \mathrm{II}}} \\
-\boldsymbol{\alpha}_{l r_{\mathrm{I}, \mathrm{II}}}^{\mathrm{T}} & \mathbf{0}
\end{array}\right]\right] \quad\left(\begin{array}{l}
\mathbf{F}_{l_{\mathrm{I}}} \\
\mathbf{F}_{l_{\mathrm{II}}}
\end{array}\right)=\mathbf{0}
\end{aligned}
$$

and

$$
\begin{gathered}
\mid\left[\begin{array}{cc}
\boldsymbol{\alpha}_{l l_{\mathrm{I}, \mathrm{I}}} & \mathbf{0} \\
\mathbf{0} & \boldsymbol{\alpha}_{l l_{\mathrm{II}, \mathrm{II}}}
\end{array}\right]-\cosh \mu\left[\begin{array}{cc}
\boldsymbol{\alpha}_{l r_{\mathrm{I}, \mathrm{I}}} & \mathbf{0} \\
\mathbf{0} & \boldsymbol{\alpha}_{l r_{\mathrm{II}, \mathrm{II}}}
\end{array}\right] \\
-\sinh \mu\left[\begin{array}{cc}
\mathbf{0} & \boldsymbol{\alpha}_{l r_{\mathrm{I}, \mathrm{II}}} \\
-\boldsymbol{\alpha}_{l r_{\mathrm{I}, \mathrm{II}}}^{T} & \mathbf{0}
\end{array}\right] \mid=0 .
\end{gathered}
$$

\section{Determination of total response of semi-infinite periodic structure}

Consider a semi-infinite periodic structure driven by an external force vector $\mathbf{F}_{0}$, e.g., the tricoupled structure in Fig. 1(a). Mead ${ }^{13}$ has shown that each wave propagation constant is associated with a particular force eigenvector, $\mathbf{F}_{l}$, which is a column vector with $n$ entries according to Eq. (4) or (16). This eigenvector was also shown to be conveniently written as

$$
\mathbf{F}_{l}=\mathbf{f} \psi,
$$

where $\mathbf{f}$ is a so-called "normalized force vector" and $\psi$ is an associated single "generalized wave coordinate." The normalized force vector specifies the relative amounts of different force components in the wave type under consideration. This force vector is obtained from Eq. (4) or (16), and it may be normalized in any desired form, but it is usually convenient to make the first element unity. While $\mathbf{f}$ depends solely on the element receptances and the considered propagation constant, then $\psi$ is also influenced by the nature of the external excitation, that is, by the contents of the $n$ elements force vector $\mathbf{F}_{0}$. Further, by analogy to Eq. (18) there is also a particular displacement vector $\mathbf{q}_{l}$ corresponding to each propagation constant; this is given by

$$
\mathbf{q}_{l}=\zeta \psi,
$$

where $\zeta$ is a so-called "normalized displacement vector," which specifies the relative amounts of different motion components in the wave type in question.

Now consider a single, positive-going wave with propagation constant $\mu_{i,+}=-\mu_{i}$, force eigenvector $\mathbf{F}_{i,+}=\mathbf{f}_{i,+} \psi_{i,+}$ and displacement vector $\mathbf{q}_{i,+}=\zeta_{i,+} \psi_{i,+}$. Substituting these into Eq. (3) yields the following relationship between the normalized force vector $\mathbf{f}_{i,+}$ and normalized displacement vector $\zeta_{i,+}$ :

$$
\boldsymbol{\zeta}_{i,+}=\boldsymbol{\alpha}_{l l} \mathbf{f}_{i,+}-\boldsymbol{\alpha}_{l r} \mathbf{f}_{i,+} e^{-\mu_{i}}
$$

If a semi-infinite periodic structure is excited at the finite left-hand end by the force vector $\mathbf{F}_{0}$, having $n$ different components, then $n$ characteristic positive-going waves are generated and these will all govern the response. The sum of the force eigenvectors corresponding to each of these waves must be in equilibrium with the applied force vector $\mathbf{F}_{0}$, that is, 


$$
\mathbf{F}_{0}=\sum_{i=1}^{n} \mathbf{F}_{i,+}=\sum_{i=1}^{n} \mathbf{f}_{i,+} \psi_{i,+}=\mathbf{f}_{+} \boldsymbol{\psi}_{+},
$$

where $\mathbf{f}_{+}$is an $n \times n$ matrix containing $n$ columns of normalized force vectors $\mathbf{f}_{i,+}$, where $i=1,2, \ldots, n$, and $\boldsymbol{\psi}_{+}$is a column vector containing $n$ generalized wave coordinates $\psi_{i,+}$, each of which is associated with a normalized force vector. The vector containing the generalized wave coordinates can now be found by a matrix inversion as

$$
\boldsymbol{\psi}_{+}=\mathbf{f}_{+}^{-1} \mathbf{F}_{0} .
$$

By analogy to Eq. (21), the total displacement vector $\mathbf{q}_{0}$ at the excitation point reads as

$$
\mathbf{q}_{0}=\sum_{i=1}^{n} \mathbf{q}_{i,+}=\sum_{i=1}^{n} \zeta_{i,+} \psi_{i,+}=\zeta_{+} \psi_{+} .
$$

The response at $j$ junctions to the right, $q_{j}$, can now be expressed as

$$
\mathbf{q}_{j}=\sum_{i=1}^{n} \mathbf{q}_{i,+} e^{-j \mu_{i}}=\sum_{i=1}^{n} \zeta_{i,+} e^{-j \mu_{i}} \psi_{i,+}=\zeta_{+} \mathbf{e}_{d}^{-\mathbf{j} \mu} \boldsymbol{\psi}_{+},
$$

where $\mathbf{e}_{d}^{-\mathbf{j} \mu}$ is an $n \times n$ diagonal matrix containing the $\left(-j \mu_{i}\right)$ 's for every wave type in the appropriate order. By using Eqs. (20) and (22), this expression finally yields

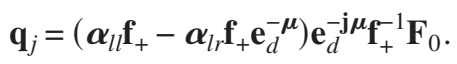

\section{ASYMMETRICALLY LOADED PERIODIC STRUCTURE}

We now consider the specific case of the asymmetrically loaded periodic structure in Fig. 1(a). According to Eq. (15), the submatrices of the periodic element's receptance matrix of this tricoupled periodic structure can be written in the partitioned form

$$
\begin{gathered}
\boldsymbol{\alpha}_{l l}=\left[\begin{array}{lll}
\alpha_{l l_{22}} & \alpha_{l l_{23}} & \alpha_{l l_{21}} \\
\alpha_{l l_{23}} & \alpha_{l l_{33}} & \alpha_{l l_{31}} \\
\alpha_{l l_{21}} & \alpha_{l l_{31}} & \alpha_{l l_{11}}
\end{array}\right], \\
\boldsymbol{\alpha}_{r r}=\left[\begin{array}{ccc}
\alpha_{l l_{22}} & -\alpha_{l l_{23}} & -\alpha_{l l_{21}} \\
-\alpha_{l l_{23}} & \alpha_{l l_{33}} & \alpha_{l l_{31}} \\
-\alpha_{l l_{21}} & \alpha_{l l_{31}} & \alpha_{l l_{11}}
\end{array}\right], \\
\boldsymbol{\alpha}_{l r}=\boldsymbol{\alpha}_{r l}^{\mathrm{T}}=\left[\begin{array}{ccc}
\alpha_{l r_{22}} & \alpha_{l r_{23}} & \alpha_{l r_{21}} \\
-\alpha_{l r_{23}} & \alpha_{l r_{33}} & \alpha_{l r_{31}} \\
-\alpha_{l r_{21}} & \alpha_{l r_{31}} & \alpha_{l r_{11}}
\end{array}\right],
\end{gathered}
$$

where subscripts 1,2, and 3 denote longitudinal, transversal, and rotational variables, respectively. These submatrices can be obtained numerically from Eqs. (9)-(13), in which the receptance submatrices of the wave-carrying column component as well as the receptance matrix of the transverse beam load components take on the forms

$$
\begin{aligned}
& \boldsymbol{\gamma}_{l l}=\left[\begin{array}{ccc}
\gamma_{l l_{22}} & \gamma_{l_{23}} & 0 \\
\gamma_{l l_{23}} & \gamma_{l_{33}} & 0 \\
0 & 0 & \gamma_{l l_{11}}
\end{array}\right], \\
& \boldsymbol{\gamma}_{r r}=\left[\begin{array}{ccc}
\gamma_{l l_{22}} & -\gamma_{l l_{23}} & 0 \\
-\gamma_{l l_{23}} & \gamma_{l_{33}} & 0 \\
0 & 0 & \gamma_{l l_{11}}
\end{array}\right], \\
& \boldsymbol{\gamma}_{l r}=\boldsymbol{\gamma}_{r l}^{T}=\left[\begin{array}{ccc}
\gamma_{l r_{22}} & \gamma_{l r_{23}} & 0 \\
-\gamma_{l r_{23}} & \gamma_{l_{33}} & 0 \\
0 & 0 & \gamma_{l r_{11}}
\end{array}\right],
\end{aligned}
$$

and

$$
\boldsymbol{\beta}=\left[\begin{array}{ccc}
\beta_{22} & 0 & 0 \\
0 & \beta_{33} & \beta_{13} \\
0 & \beta_{13} & \beta_{11}
\end{array}\right] .
$$

Closed form expressions of all the individual component receptances in Eqs. (27) and (28) may be found in Ref. 15. In the present analysis all components are modeled by using Bernoulli-Euler beam theory, albeit with a correction for shear deformation, ${ }^{16}$ which is required especially for obtaining correct natural frequencies of the periodic element in the considered frequency range. Note that it is the receptance $\beta_{13}$, which is solely responsible for the coupling between longitudinal and flexural wave motions in this type of periodic structure. Receptance $\beta_{13}$, of course, becomes an integral part of $\alpha_{l l_{21}}, \alpha_{l l_{31}}, \alpha_{l r_{21}}$, and $\alpha_{l r_{31}}$ when these receptances of Eq. (26) are computed from Eqs. (10) and (11).

Having derived expressions for the receptances, we are now able to determine the propagation constants for the possible wave types in the periodic structure and, subsequently, the total response that they generate. Propagation constants are determined from Eq. (17), and for the considered tricoupled system this yields

$$
\begin{gathered}
\mid\left[\begin{array}{ccc}
\alpha_{l l_{22}} & 0 & 0 \\
0 & \alpha_{l l_{33}} & \alpha_{l l_{31}} \\
0 & \alpha_{l l_{31}} & \alpha_{l l_{11}}
\end{array}\right]-\cosh \mu\left[\begin{array}{ccc}
\alpha_{l r_{22}} & 0 & 0 \\
0 & \alpha_{l r_{33}} & \alpha_{l r_{31}} \\
0 & \alpha_{l r_{31}} & \alpha_{l r_{11}}
\end{array}\right] \\
-\sinh \mu\left[\begin{array}{ccc}
0 & \alpha_{l r_{23}} & \alpha_{l r_{21}} \\
-\alpha_{l r_{23}} & 0 & 0 \\
-\alpha_{l r_{21}} & 0 & 0
\end{array}\right] \mid=0 .
\end{gathered}
$$

From this equation three pairs of propagation constants $\pm \mu_{i}$ are found, where $i=A, B, C$. Due to wave conversion, all three wave types contain contributions of axial, transversal, and rotational motions, and the total response of the semiinfinite periodic structure of Fig. 1(a) is thus the sum of contributions from the three, positive-going waves. Hence, from Eq. (25) we obtain the total response at the $j$ th junction; when written in full this yields

L. Friis and M. Ohlrich: Coupled longitudinal-flexural wave motion 


$$
\begin{aligned}
\left(\begin{array}{l}
q_{j_{2}} \\
q_{j_{3}} \\
q_{j_{1}}
\end{array}\right)= & \left(\left[\begin{array}{lll}
\alpha_{l l_{22}} & \alpha_{l l_{23}} & \alpha_{l l_{21}} \\
\alpha_{l l_{23}} & \alpha_{l l_{33}} & \alpha_{l l_{31}} \\
\alpha_{l l_{21}} & \alpha_{l l_{31}} & \alpha_{l l_{11}}
\end{array}\right] \mathbf{f}_{+}-\left[\begin{array}{ccc}
\alpha_{l r_{22}} & \alpha_{l r_{23}} & \alpha_{l r_{21}} \\
-\alpha_{l r_{23}} & \alpha_{l r_{33}} & \alpha_{l r_{31}} \\
-\alpha_{l r_{21}} & \alpha_{l r_{31}} & \alpha_{l r_{11}}
\end{array}\right]\right. \\
& \left.\times \mathbf{f}_{+}\left(\begin{array}{ccc}
e^{-\mu_{A}} & 0 & 0 \\
0 & e^{-\mu_{B}} & 0 \\
0 & 0 & e^{-\mu_{C}}
\end{array}\right]\right)\left[\begin{array}{ccc}
e^{-j \mu_{A}} & 0 & 0 \\
0 & e^{-j \mu_{B}} & 0 \\
0 & 0 & e^{-j \mu_{C}}
\end{array}\right] \\
& \times \mathbf{f}_{+}^{-1}\left(\begin{array}{l}
F_{0_{2}} \\
F_{0_{3}} \\
F_{0_{1}}
\end{array}\right),
\end{aligned}
$$

where the matrix $\mathbf{f}_{+}$containing the three normalized force vectors $\mathbf{f}_{i,+}$ is given as

$$
\mathbf{f}_{+}=\left[\begin{array}{lll}
\mathbf{f}_{A,+} & \mathbf{f}_{B,+} & \mathbf{f}_{C,+}
\end{array}\right]=\left[\begin{array}{ccc}
1 & 1 & 1 \\
X_{A,+} & X_{B,+}{ }_{32} & X_{C,+} \\
X_{A,+} & X_{B,+} & X_{C,+}{ }_{12}
\end{array}\right] .
$$

Here $X_{i,+_{32}}$ is the ratio between excitations from moment and transversal force and $X_{i,+}$ is the ratio between axial force and transversal force; these force ratios for the $i$ th wave are derived in Appendix A.

Now, in order to explain why each wave may contribute differently to the total response, it is necessary to quantify the relative amounts of flexural and longitudinal wave motion contained in each wave type. As shown in Ref. 7, this can be done by introducing the ratio $E_{\mathrm{kin}, F} / E_{\mathrm{kin}, L}$, which expresses the ratio between the maximum kinetic energies of flexural and longitudinal motion in the wave-carrying column component. In order to find these energies it is therefore necessary to determine the longitudinal and transversal motions at all positions in the column component caused by a single wave. Expressions for these "interior" motions are derived in Appendix B in terms of the displacements at the element's left-hand end and the propagation constant of the wave type considered. Note that any second-order contributions, say, transverse motion in quasilongitudinal waves and longitudinal motion in flexural waves are neglected in the calculations, and so is the rotational part of the flexural kinetic energy. Now, for the $i$ th wave, the junction displacements at the left-hand end are found from the $i$ th term in the series Eq. (24), giving

$$
\begin{aligned}
& {\left[\begin{array}{l}
q_{i, j,+_{2}} \\
q_{i, j,+_{3}} \\
q_{i, j,+_{1}}
\end{array}\right]=\left[\begin{array}{c}
\zeta_{i,+_{2}} \\
\zeta_{i,+_{3}} \\
\zeta_{i,+}
\end{array}\right] e^{-j \mu_{i}} \psi_{i,+}} \\
& =\left(\left[\begin{array}{lll}
\alpha_{l l_{22}} & \alpha_{l l_{23}} & \alpha_{l l_{21}} \\
\alpha_{l l_{23}} & \alpha_{l l_{33}} & \alpha_{l l_{31}} \\
\alpha_{l l_{21}} & \alpha_{l l_{31}} & \alpha_{l l_{11}}
\end{array}\right]\left(\begin{array}{c}
1 \\
X_{A,+} \\
X_{A,+}
\end{array}\right)\right. \\
& \left.-\left[\begin{array}{ccc}
\alpha_{l r_{22}} & \alpha_{l r_{23}} & \alpha_{l r_{21}} \\
-\alpha_{l r_{23}} & \alpha_{l r_{33}} & \alpha_{l r_{31}} \\
-\alpha_{l r_{21}} & \alpha_{l r_{31}} & \alpha_{l r_{11}}
\end{array}\right]\left(\begin{array}{c}
1 \\
X_{A,+} \\
X_{A,+}
\end{array}\right) e^{-\mu_{i}}\right) e^{-j \mu_{i}} \psi_{i,+} .
\end{aligned}
$$

Notice that all three junction displacements of the considered periodic element are proportional to $\left(e^{-j \mu_{i}} \psi_{i,+}\right)$, and so are also the displacements in the column component. However, $\left(e^{-j \mu_{i}} \psi_{i,+}\right)$ cancels out in the expression for the energy ratio $E_{\mathrm{kin}, F} / E_{\mathrm{kin}, L}$ since both energies are proportional to the square of the column displacements. This implies that the energy ratio only depends upon the normalized displacements in the vector $\zeta_{i,+}$ and not on the actual displacements, which additionally depend upon position $j$ and the external excitation of the periodic structure.

\section{NUMERICAL INVESTIGATION AND DISCUSSION}

Numerical analyses and parameter studies have been conducted for both asymmetric and symmetric types of semiinfinite periodic structures in order to examine and reveal the effects of wave conversion. In both types of structures the mass and natural frequencies of the transverse beam components are taken to be identical in order to facilitate a direct comparison of the system's properties. The results presented herein are for relatively short beam loads that are tuned to have their fundamental natural frequency well within the considered frequency range. The purpose of this parameter choice is to illustrate clearly the different and complicated wave conversion phenomena that take place. All computations and matrix manipulations have been done using MATLAB, version 6.5.

Apart from the above-mentioned dimensions of the beam loads, the structural properties and dimensions of the element components used in these simulations are similar to those used in an experimental investigation, being presented in a companion paper. ${ }^{14}$ The material is acrylic with Young's modulus $E=5.4 \times 10^{9} \mathrm{~N} / \mathrm{m}^{2}$ and a density of $\rho$ $=1200 \mathrm{~kg} / \mathrm{m}^{3}$. Material damping is modeled by letting Young's modulus become complex as $\underline{E}=E(1+i \eta)$, where $\eta$ is the damping loss factor. In the simulations, an arbitrarily low value of $\eta=0.001$ has been chosen in order to clearly reveal the wave coupling phenomena. The dimensions of the wave-carrying column component are length $l=235 \mathrm{~mm}$, thickness $h=15 \mathrm{~mm}$, and width $b=20 \mathrm{~mm}$, whereas the transverse load beam has length $l_{t}=l / 8$, thickness parameter $h_{t}=h$, and width parameter $b_{t}=2 b$. Results herein are presented as a function of the nondimensional frequency parameter $\Omega=\left(k_{b} l\right)^{2}$, where $k_{b}$ is the wave number for free flexural waves in the column component. Substituting the bending stiffness of the column yields

$$
\Omega=\left(k_{b} l\right)^{2}=\omega(12 \rho / E)^{1 / 2}\left(l^{2} / h\right) .
$$

\section{A. Propagation constants}

It is recalled that in the simpler case of a symmetrically loaded structure, ${ }^{4,5}$ there is no longitudinal-flexural wave coupling, and the response is therefore governed by a purely longitudinal wave type and by two purely flexural wave types. In other words, flexural waves cannot be excited by a longitudinal excitation force, and vice versa. By contrast, all three wave types are coupled in the case of an asymmetrically loaded structure; these waves (and the resulting response) thus include contributions of longitudinal and flex- 

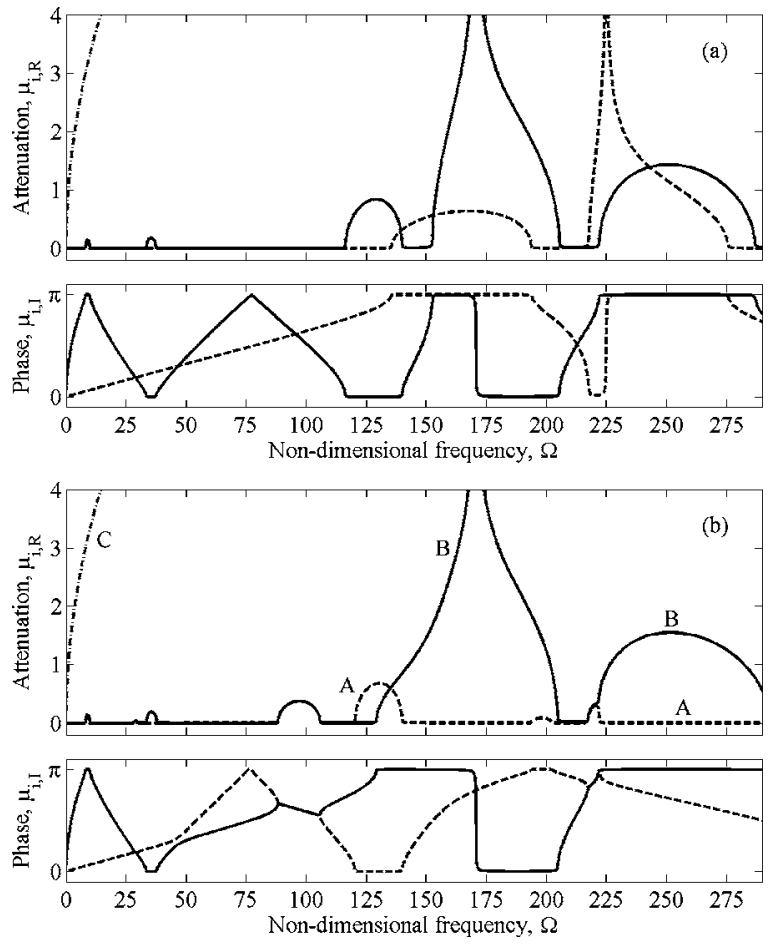

FIG. 3. Frequency variation of the real and imaginary parts of the propagation constants $\mu_{i}=\mu_{i, R}+i \mu_{i, I}$ for the three wave types. (a) Symmetrically loaded column-structure: - - -, longitudinal wave; - flexural wave; ---, flexural near field. (b) Asymmetrically loaded column-structure: - - -, flexural-longitudinal wave A; - , flexural-longitudinal wave B; ---, primarily flexural near-field C. Loss factor $\eta=0.001$.

ural wave motions, i.e., all three wave types as well as the total response contain contributions from longitudinal (axial), transverse, and rotational displacements. The wave characteristics for both types of structures are presented in Fig. 3, which shows the frequency variation of the real and imaginary parts of the propagation constants associated with the three wave types.

Results for the symmetrically loaded column structure are shown in Fig. 3(a). The longitudinal wave motion, which is governed by a single characteristic wave, is seen to propagate (without attenuation in wave amplitude) over a broadband of frequencies up to $\Omega=135$, and with a phase change per element, $\mu_{I}$, that is almost proportional to $\Omega$. From there on, the transmission characteristics for longitudinal waves exhibit both smooth and resonant attenuation zones with intermediate zones of propagation. The frequency position and "bandwidth" of the resonant attenuation zone(s) is controlled by the mass, length, and modal characteristic of the load components; the peak attenuation thus occurs at a frequency at which the transverse beam-load components vibrate in a virtually midpoint-fixed, symmetrical mode. For clarity we have chosen a system with relatively short beam components that has only a single resonant attenuation zone within the considered frequency range. (For a system with somewhat longer beam components, say $l_{t}=0.8 l$, there are four resonant attenuation zones ${ }^{4,5}$ in the considered frequency range, and similarly four resonant attenuation zones for flexural wave motion). Such resonant attenuation zones are bounded by the natural frequencies of a single periodic element with both ends free. The magnitude and bandwidth of the smooth at- tenuation zone is controlled by the modal mass of the load components. The lower bounding frequency of a smooth attenuation zone is identical to the natural frequency of a single element with both ends free, whereas the upper bounding frequency is identical with the natural frequency of a single element with both ends fixed.

The flexural wave motion is governed by two waves, of which one is associated with a very high attenuation constant and can be regarded as a near field. For the sake of simplicity the phase constant of this wave has been omitted in Fig. 3(a). The other flexural wave also has the pattern of smooth and resonant attenuation zones. Apart from two very weak attenuation zones, this flexural wave is seen to propagate up until $\Omega=115$. At the peak attenuation frequency there is virtually neither rotation nor transverse displacement at the column-beam junctions. This frequency is related to the natural frequency of the midpoint-fixed mode of the transverse beam components that vibrate in an antisymmetric mode as opposed to the symmetric mode at the same frequency that governs the longitudinal resonant attenuation zones. However, peak attenuation of the flexural wave occurs at a somewhat lower frequency. This shift is caused by a minimum in $\alpha_{l l_{23}}=\alpha_{l l_{32}}$ at an even lower frequency, which creates zero transverse displacement due to moment excitation and zero rotation due to transverse force excitation. Further, at the frequency of peak attenuation the two flexural waves become complex conjugates. It is well known ${ }^{4}$ that the lower bounding frequencies of smooth flexural attenuation zones are controlled by element modes of zero rotation at the junctions, whereas all other bounding frequencies of attenuation zones are controlled by element modes with zero transverse displacements.

We now consider the results in Fig. 3(b) for the asymmetrically loaded periodic structure. Due to longitudinalflexural wave coupling, all three wave types contain axial, transversal, and rotational motions. The denoted wave type $\mathrm{C}$, however, is again associated with a very large attenuation constant, and it can be regarded primarily as a flexural nearfield, as will be revealed in the next section. Apart from the gross overall resemblance with the results for the symmetric structure, a number of important differences can be observed regarding the propagation constants of the remaining two wave types in the asymmetric structure, say, waves A and B.

From the phase constants, it is seen that the two curves do not cross over in a straightforward manner, as was the case in Fig. 3(a). Instead the curves either (i) diverge from one another or (ii) they merge, so that the propagation constants almost become complex conjugates with a nonzero attenuation constant. The first phenomenon is seen to occur at $\Omega=46$; at this frequency the phase constant of the apparently longitudinal-type wave, A, change over to a steeper phase characteristic that is usually associated with a flexuraltype wave. This appears to be a clear indicator of the occurrence of a strong wave coupling or wave conversion. The second phenomenon of strong wave coupling is seen to occur in the bands of $\Omega=28$ to 30,88 to 106 and 217 to 222 , in which the coupled longitudinal-flexural waves are also attenuated as they progress through the periodic structure. Such complex conjugate zones, or "amber bands," have also 


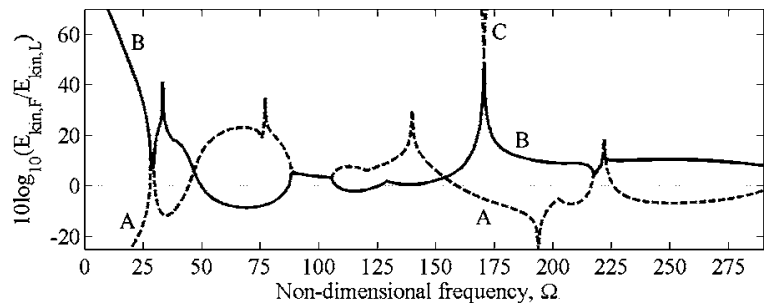

FIG. 4. Energy ratios $E_{\mathrm{kin}, F} / E_{\mathrm{kin}, L}$ for wave types in an asymmetrically loaded column structure. - - -, flexural-longitudinal wave A; - , flexurallongitudinal wave B; ---, primarily flexural near-field C. Loss factor $\eta$ $=0.001$.

been examined in Refs. 17,18. It should be mentioned that these two types of coupling phenomena occur most strongly in lightly damped systems, whereas they may disappear more or less completely in systems with high structural damping. Mead and Markus ${ }^{7}$ observed this in their study of a simply loaded periodic system.

From the attenuation constants in Fig. 3(b) it is seen that only a single resonant attenuation zone is present and that this is associated with wave type B, whereas wave type A has a full propagation zone in more or less the same frequency band. The peak attenuation is noted to occur at a frequency of $\Omega=170$, which is nearly the same as for the symmetric system in Fig. 3(a). Another important observation is that the coupling enforces a merging of the previously uncoupled, flexural resonant attenuation zone and the smooth longitudinal attenuation zone into a single attenuation zone. A parameter study reveals that this merging generally takes place when the two types of attenuation zones (in the uncoupled symmetric case) appear close to one another or are actually coinciding.

What remains is a characterization of the type of wave motions that occur in the different frequency bands. The propagation constants presented in Fig. 3(b) do not solely facilitate such a classification of the actual wave nature, but this can be revealed by computing the normalized displacement vectors, $\boldsymbol{\zeta}_{i,+}$, as given by Eq. (32).

\section{B. Nature of wave types}

The relative amounts of flexural and longitudinal motions contained in each wave type can be quantified by the kinetic energy ratio $\left(E_{\mathrm{kin}, F} / E_{\mathrm{kin}, L}\right)$, which was defined in Sec. IV. This quantity has been calculated numerically for each wave type in terms of the normalized displacement vector, $\zeta_{i,+}$ and propagation constant $\mu_{i,+}=-\mu_{i}$ for its positive-going wave.

Figure 4 show such results in the form of energy ratio levels for the three wave types in the asymmetrically loaded column structure; corresponding propagation constants are given in Fig. 3(b). At low frequencies a distinction between the wave types is readily made. Wave type A is clearly longitudinal and wave type $\mathrm{B}$ is clearly flexural, whereas wave type $\mathrm{C}$ is a flexural near-field with an energy ratio level exceeding $70 \mathrm{~dB}$ ! This behavior of type A and B waves extends up until about $\Omega=27$. From there on both wave types must be classified as being longitudinal-flexural as each of them alternates in a complicated manner between being predomi-

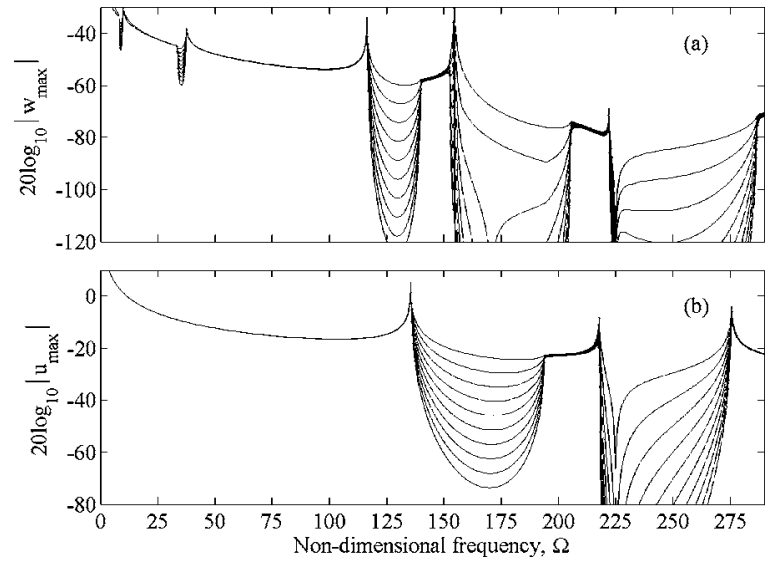

FIG. 5. Maximum displacement amplitudes in the column component of the first ten elements of the semi-infinite, symmetrically loaded structure. (a) Maximum transverse displacement $\left|w_{\max }\right|$ of flexural motion generated by an external harmonic moment $M_{0}$. (b) Maximum longitudinal displacement $\left|u_{\max }\right|$ generated by an external harmonic force $F_{0}$. Loss factor $\eta=0.001$.

nantly longitudinal and predominantly flexural, or fully mixed, with approximately equal contributions. In complex conjugate zones the relative amount of flexural and longitudinal motions in the two mixed wave types are seen to be of the same order of magnitude. Further, wave types B and C become complex conjugate at $\Omega=170$, at an energy level of about $40 \mathrm{~dB}$. At some frequencies (around $\Omega=46$ and 154) and in complex conjugate zones both of the two mixed wave types are predominantly flexural.

\section{Response of semi-infinite periodic structures}

Finally, we shall demonstrate the effect of wave coupling on the response of semi-infinite periodic structures to external point excitation. Two types of external harmonic excitations are considered, namely a nondimensional moment of amplitude $M_{0}=(E I) / l$, and a nondimensional longitudinal (axial) force of amplitude $F_{0}=E S$, where $I$ is the second moment of area of the column component and $S$ is its cross-sectional area. Again, the results for a symmetrically loaded structure are first shown in order to clearly demonstrate what happens when the asymmetrically loaded structure is considered.

For the symmetrically loaded system Fig. 5 shows the maximum displacements in the first ten column components in terms of the transverse displacement $\left|w_{\max }\right|$ in the uncoupled flexural wave motion and the longitudinal displacement $\left|u_{\max }\right|$ in the purely longitudinal wave motion. These interior, maximum responses of the column components are calculated from the expressions in Appendix B by using the displacements for each wave type at the left-hand end of the considered element, as given in Eq. (32). Figure 5 clearly shows the occurrence and effect of the pass bands and stop bands that are associated with the corresponding propagation constants in Fig. 3(a). Further, it is seen that the system responds strongly at the bounding frequencies, which are governed by the natural frequencies of a single periodic element.

In the asymmetrically loaded system [see Fig. 1(a)] the harmonic response results from a combination of contributions from the coupled positive-going waves. The signifi- 

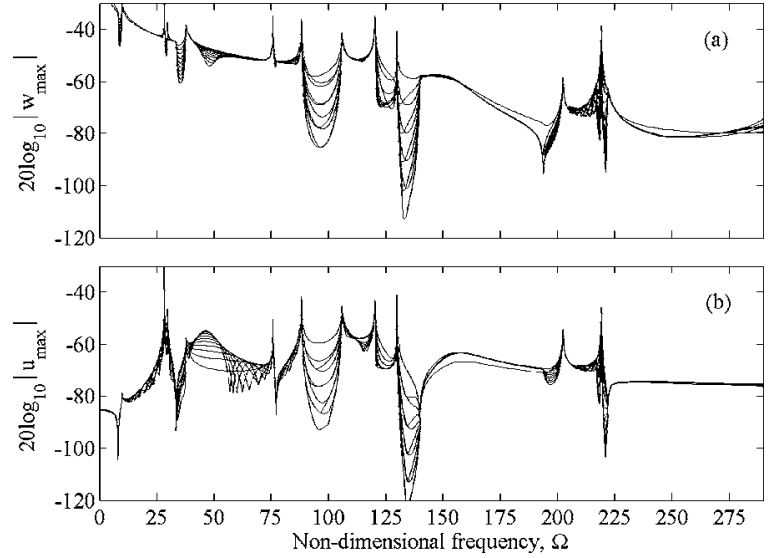

FIG. 6. Maximum displacements in the column component of the first ten elements of a semi-infinite, asymmetrically loaded structure, excited by an external harmonic moment $M_{0}$. (a) Maximum transverse displacement $\left|w_{\max }\right|$ of flexural motion; (b) Maximum longitudinal displacement $\left|u_{\max }\right|$. Loss factor $\eta=0.001$.

cance and contribution of each wave with respect to the total response does not only depend upon the type of external excitation, but also on the energy ratios and the propagation constants. This implies that the calculated displacement responses can be explained from the results in Fig. 3(b) and Fig. 4. The maximum displacement responses for the case of the asymmetrically loaded periodic system driven by an external harmonic moment $M_{0}$ are shown in Fig. 6. Compared to the symmetrically loaded system, it is seen that the wave coupling has a drastic effect; with the exception of a few narrow stop bands, the waves are seen to propagate unattenuated in most parts of the considered frequency range. Thus, in the bands from $\Omega=140$ to 205 and from $\Omega=222$ and up, in which the type B wave is predominantly flexural (see Fig. 4), the strong attenuation zones have no significant effect on either of the groups of responses in Fig. 6. The reason for this is that the propagating type-A wave is dominating, even though it is predominantly longitudinal. Generally, this is found to be the case whenever one of the wave types is propagating and the other wave type is strongly attenuated. A close inspection further reveals that for most frequencies exceeding $\Omega=165$, the longitudinal displacements in Fig. 6(b) are even higher than the transverse flexural displacements in Fig. 6(a). It is only at low frequencies, below $\Omega \approx 25$, that longitudinal displacement responses are insignificant. From $\Omega=30$ to 88 the longitudinal displacements are about $13 \mathrm{~dB}$ lower than the transverse displacements, and they become very similar in the range from $\Omega=88$ to 165 .

Moreover, an appreciable but uneven attenuation from element to element is noted to occur in narrow frequency zones where wave types A and B are both governed by appreciable attenuation constants; see $\Omega \approx 95$ and 135 in Fig. 3(b) and Fig. 6. Small attenuation also occurs when the two wave types are both propagating, and their contributions of transverse and longitudinal displacements are of the same order of magnitude. When superimposed the waves cancel one another either partly or completely. This is seen to be the case in the frequency range from $\Omega=38$ to 75 in Fig. 6 .

Results for harmonic excitation with an external longi-

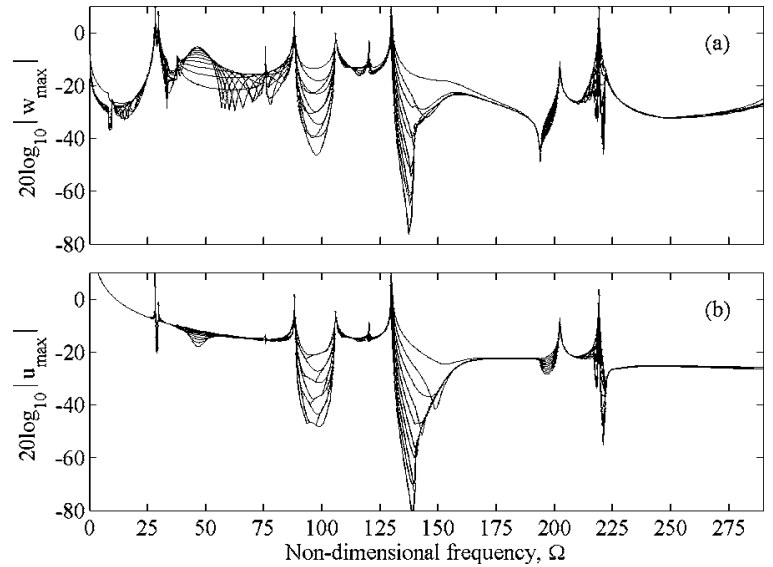

FIG. 7. The same as in Fig. 6, but for an external harmonic axial force excitation $F_{0}$.

tudinal (axial) force $F_{0}$ are shown in Fig. 7. From Fig. 7(b) it is seen again that the wave coupling has a very strong effect when compared to the results for the uncoupled longitudinal responses in Fig. 5(b). Overall the results are very similar to those in Fig. 6 , although the stop band at $\Omega \approx 135$ is slightly wider. Apart from the stop bands and the peaks at some of the bounding frequencies, the longitudinal displacement responses in Fig. 7(b) may readily be shown to follow closely the asymptotic response of a simple continuous and semiinfinite column structure of the same cross-sectional area $S$. This means that the corresponding longitudinal velocity responses are grossly independent of frequency in the considered frequency range. Very similar are also the spectral shapes of the system's "cross-properties," that is, the transverse flexural displacements in the column elements generated by the longitudinal force $F_{0}$ [see Fig. $\left.7(\mathrm{a})\right]$ and the longitudinal displacements generated by the moment excitation $M_{0}$; see Fig. 6(b). In the case of longitudinal force excitation it is the transverse displacement that is insignificant at the low frequencies below $\Omega=27$. From $\Omega=30$ to 165 the longitudinal and transverse displacements are very similar in amplitude, and from there on the longitudinal displacement is about $6 \mathrm{~dB}$ higher than the transverse, as was the case with moment excitation.

\section{CONCLUSIONS}

A new model based on composite element receptances has been developed for studying phenomena of flexurallongitudinal wave coupling in fully tricoupled periodic waveguides. Specifically examined are lightly damped periodic structures with multiple and resonant side branches in the form of offset transverse beams attached at regular intervals. This may resemble a one-dimensional model of column-beam skeletons in buildings or idealized plane-wave, normal incidence models of web-stiffened panels, ship hulls with decks to one side only, etc. Computed results of complex propagation constants, which govern the wave fields in periodic structures, have clearly revealed pass and stop band characteristics and wave coupling phenomena, which are shown to enhance the long-range transmission when the periodic structure is unconstrained and hence allowed to vi- 
brate freely in its plane. This system can support three coupled wave types, and their importance is clarified and quantified from computations of their contribution to ratios of maximum flexural and longitudinal kinetic energies in the wave-carrying components. For this asymmetrically pointloaded periodic structure it is found that two wave types can be categorized as being coupled flexural-longitudinal waves in major parts of the considered frequency range, because both types alternate in a complicated manner between being either fully mixed or occasionally predominantly longitudinal or predominantly flexural. The remaining third wave type is a flexural type "near-field" as is the case for a corresponding, but symmetrical system in which longitudinal and flexural wave types are uncoupled.

The determined distribution of displacements in each wave type is furthermore used for calculating the spatial variation of the responses of a semi-infinite periodic structure that is subjected to end-point harmonic excitations by either a longitudinal force or by a moment. In contrast to the corresponding uncoupled symmetrical system with significant broadband attenuation of flexural-type waves, the numerical results show that the flexural-longitudinal wave coupling in a system with resonant side branches has a drastic effect on the wave propagation properties. Despite the system is excited by an external moment - and one of the two wave types is predominantly flexural with a broad stop band - it is unexpectedly found that the inherent wave coupling results in a highly enhanced wave transmission with very little attenuation of flexural motion from element to element. This enhanced transmission is caused by the other wave type, which is predominantly longitudinal and propagates with significant components of both flexural and longitudinal displacements. Also in the case of longitudinal force excitation, wave propagation similarly occurs at most frequencies and both types of response displacements are of the same order of magnitude. For the structural components and dimensions considered in this study the longitudinal responses along the periodic structure are furthermore found to be only little influenced by the transverse beam loads; hence, responses follow grossly the asymptotic response of a simple continuous waveguide of the same cross-sectional area. With the present composite-receptance approach other types of structural side-branches components or other boundary conditions can easily be incorporated in the developed prediction model.

\section{APPENDIX A: DERIVATION OF FORCE RATIOS FOR THE iTH WAVE}

For a characteristic positive-going wave traveling through the periodic system with propagation constant $-\mu_{i}$, the corresponding force eigenvector can be found from Eq. (16). For the tricoupled structure this yields

$$
\left[\begin{array}{ccc}
\alpha_{l l_{22}}-\alpha_{l r_{22}} \cosh \mu_{i} & -\alpha_{l r_{23}} \sinh \mu_{i} & -\alpha_{l r_{21}} \sinh \mu_{i} \\
\alpha_{l r_{23}} \sinh \mu_{i} & \alpha_{l l_{33}}-\alpha_{l r_{33}} \cosh \mu_{i} & \alpha_{l l_{31}}-\alpha_{l r_{31}} \cosh \mu_{i} \\
\alpha_{l r_{21}} \sinh \mu_{i} & \alpha_{l l_{31}}-\alpha_{l r_{31}} \cosh \mu_{i} & \alpha_{l l_{11}}-\alpha_{l r_{11}} \cosh \mu_{i}
\end{array}\right]\left(\begin{array}{c}
F_{i,+} \\
F_{i,+3} \\
F_{i,+}
\end{array}\right)=\left(\begin{array}{c}
0 \\
0 \\
0
\end{array}\right) \text {. }
$$

The first and second of these equations can be written as

$$
\begin{aligned}
& \left(\alpha_{l l_{22}}-\alpha_{l r_{22}} \cosh \mu_{i}\right) F_{i,+_{2}}-\left(\alpha_{l r_{23}} \sinh \mu_{i}\right) F_{i,+_{3}}-\left(\alpha_{l r_{21}} \sinh \mu_{i}\right) F_{i,+_{1}}=0, \\
& \left(\alpha_{l r_{23}} \sinh \mu_{i}\right) F_{i,+_{2}}+\left(\alpha_{l l_{33}}-\alpha_{l r_{33}} \cosh \mu_{i}\right) F_{i,+_{3}}+\left(\alpha_{l l_{31}}-\alpha_{l r_{31}} \cosh \mu_{i}\right) F_{i,+_{1}}=0 .
\end{aligned}
$$

By multiplying the first equation with $\left(\alpha_{l l_{31}}-\alpha_{l r_{33}} \cosh \mu_{i}\right)$ and the second equation with $\left(\alpha_{l r_{21}}\right.$ sinh $\left.\mu_{i}\right)$, and finally subtracting the two, $F_{i,+}$ is eliminated:

$$
\begin{aligned}
& \left(\alpha_{l l_{22}}-\alpha_{l r_{22}} \cosh \mu_{i}\right)\left(\alpha_{l l_{31}}-\alpha_{l r_{31}} \cosh \mu_{i}\right) F_{i,+_{2}}-\left(\alpha_{l r_{23}} \sinh \mu_{i}\right)\left(\alpha_{l l_{31}}-\alpha_{l r_{31}} \cosh \mu_{i}\right) F_{i,+_{3}}+\left(\alpha_{l r_{23}} \sinh \mu_{i}\right)\left(\alpha_{l r_{21}} \sinh \mu_{i}\right) F_{i,+_{2}} \\
& \quad+\left(\alpha_{l l_{33}}-\alpha_{l r_{33}} \cosh \mu_{i}\right)\left(\alpha_{l r_{21}} \sinh \mu_{i}\right) F_{i,+_{3}}=0 .
\end{aligned}
$$

From this the ratio between $F_{i,+}$ and $F_{i,+_{2}}$ can be found as

$$
X_{i,+_{32}}=\frac{F_{i,+_{3}}}{F_{i,+_{2}}}=-\frac{\left(\alpha_{l l_{22}}-\alpha_{l r_{22}} \cosh \mu_{i}\right)\left(\alpha_{l l_{31}}-\alpha_{l r_{31}} \cosh \mu_{i}\right)+\left(\alpha_{l r_{23}} \sinh \mu_{i}\right)\left(\alpha_{l r_{21}} \sinh \mu_{i}\right)}{\left(\alpha_{l l_{33}}-\alpha_{l r_{33}} \cosh \mu_{i}\right)\left(\alpha_{l r_{21}} \sinh \mu_{i}\right)-\left(\alpha_{l r_{23}} \sinh \mu_{i}\right)\left(\alpha_{l l_{31}}-\alpha_{l r_{31}} \cosh \mu_{i}\right)} \text {. }
$$

The use of any other two equations from the matrix in Eq. (A1) would result in the same ratio. The ratio between $F_{i,+}$ and $F_{i,++_{2}}$ is found likewise by multiplying the first equation with $\left(\alpha_{l l_{33}}-\alpha_{l r_{33}} \cosh \mu_{i}\right)$ and the second equation with $\left(-\alpha_{l r_{23}} \sinh \mu_{i}\right)$, and further subtracting the two in order to eliminate $F_{i,+}$. This yields

$$
X_{i,+_{12}}=\frac{F_{i,+_{1}}}{F_{i,+_{2}}}=-\frac{\left(\alpha_{l l_{22}}-\alpha_{l r_{22}} \cosh \mu_{i}\right)\left(\alpha_{l l_{33}}-\alpha_{l r_{33}} \cosh \mu_{i}\right)+\left(\alpha_{l r_{23}} \sinh \mu_{i}\right)\left(\alpha_{l r_{23}} \sinh \mu_{i}\right)}{\left(\alpha_{l l_{31}}-\alpha_{l r_{31}} \cosh \mu_{i}\right)\left(\alpha_{l r_{23}} \sinh \mu_{i}\right)-\left(\alpha_{l r_{21}} \sinh \mu_{i}\right)\left(\alpha_{l l_{33}}-\alpha_{l r_{33}} \cosh \mu_{i}\right)} .
$$




\section{APPENDIX B: LONGITUDINAL AND TRANSVERSAL RESPONSES IN THE COLUMN COMPONENT}

The response at an arbitrary position $x$ in a column component or in a transverse beam component can be obtained in terms of the displacements and rotations at the ends of the periodic element. The longitudinal displacements, transverse displacements, and rotations at the left- and right-hand ends of an element due to the $i$ th wave type are now denoted $u_{l, i}$, $u_{r, i}, w_{l, i}, w_{r, i}, \varphi_{l, i}$, and $\varphi_{r, i}$, respectively. Index $l$ and $r$ refer to left- and right-hand end. Thus, for a single harmonic wave type with propagation constant $-\mu_{i}$, the complex displacement amplitudes at the right- and left-hand ends of the periodic element are related as

$$
u_{r, i}=u_{l, i} e^{-\mu_{i}}, \quad w_{r, i}=w_{l, i} e^{-\mu_{i}}, \quad \text { and } \quad \varphi_{r, i}=\varphi_{l, i} e^{-\mu_{i}} .
$$

Utilizing these relationships, we can express the displacements in the column component in terms of the element's displacements at the left-hand end and the propagation constant for the wave considered. The displacements at the lefthand end of the $j$ th element are given in Eq. (32); hence

$$
u_{l, i}=q_{i, j,+}, \quad w_{l, i}=q_{i, j,+_{2}} \quad \text { and } \quad \varphi_{l, i}=q_{i, j,+_{3}} .
$$

By using general wave theory, ${ }^{1}$ the local $x$ dependence of the longitudinal displacement $u_{i}(x)$ in the column component, due to the $i$ th wave, takes the form

$$
u_{i}(x)=A_{i} \cos k_{l} x+B_{i} \sin k_{l} x,
$$

where $k_{l}$ is the wave number for free longitudinal waves. Applying Eq. (B1), this can be expressed as

$$
u_{i}(x)=u_{l, i}\left[\cos k_{l} x+\sin k_{l} x\left(e^{-\mu_{i}}-\cos k_{l} l\right) / \sin k_{l} l\right] .
$$

In a similar form the transverse displacement $w_{i}(x)$ in the column component due to the $i$ th wave is given by

$$
\begin{aligned}
w_{i}(x)= & a_{i}\left(\cos k_{b} x-\cosh k_{b} x\right)+b_{i}\left(\sin k_{b} x-\sinh k_{b} x\right) \\
& +w_{l, i} \cosh \left(k_{b} x\right)+\left(\varphi_{l, i} / k_{b}\right) \sinh \left(k_{b} x\right),
\end{aligned}
$$

and by applying Eq. (B1), $a_{i}$ and $b_{i}$ are expressed as

$$
\begin{aligned}
& a_{i}=\left[\left[w_{l, i}\left(\cosh k_{b} l \cos k_{b} l-\sinh k_{b} l \sin k_{b} l-1\right)\right.\right.
\end{aligned}
$$

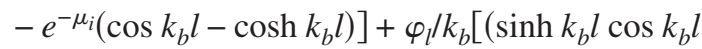

$$
\begin{aligned}
& \left.-\cosh k_{b} l \sin k_{b} l\right)+e^{-\mu_{i}\left(\sin k_{b} l\right.} \\
& \left.\left.\left.-\sinh k_{b} l\right)\right]\right] /\left[2\left(\cos k_{b} l \cosh k_{b} l-1\right)\right]
\end{aligned}
$$

and

$$
\begin{aligned}
b_{i}= & {\left[a_{i}\left(\cos k_{b} l-\cosh k_{b} l\right)+w_{l}\left(\cosh k_{b} l-e^{-\mu_{i}}\right)\right.} \\
& \left.+\varphi_{l} / k_{b} \sinh k_{b} l\right] /\left[\sinh k_{b} l-\sin k_{b} l\right],
\end{aligned}
$$

where $k_{b}$ is the wave number for free flexural waves.

According to Eq. (24), the total response is a sum of response contributions from all three positive-going waves and by using linear superposition, the longitudinal displacement $u(x)$ and the transverse displacement $w(x)$ in the column yield

$$
\begin{aligned}
u(x) & =\sum_{i=A}^{C} u_{i}(x) \\
& =\sum_{i=A}^{C} u_{l, i}\left[\cos k_{l} x+\sin k_{l} x\left(e^{-\mu_{i}}-\cos k_{l} l\right) / \sin k_{l} l\right]
\end{aligned}
$$

and

$$
\begin{aligned}
w(x)= & \sum_{i=A}^{C} w_{i}(x) \\
= & \sum_{i=A}^{C}\left[a_{i}\left(\cos k_{b} x-\cosh k_{b} x\right)+b_{i}\left(\sin k_{b} x-\sinh k_{b} x\right)\right. \\
& \left.+w_{l, i} \cosh \left(k_{b} x\right)+\left(\varphi_{l, i} / k_{b}\right) \sinh \left(k_{b} x\right)\right] .
\end{aligned}
$$

${ }^{1}$ L. Cremer and H. O. Leilich, "Zur theorie der Biegekettenleiter" ("On theory of flexural periodic systems"), Arch. Elektr. Uebertrag. 7, 261-270 (1953).

${ }^{2}$ L. Cremer, M. Heckl, and E. E. Ungar, Structure-Borne Sound (SpringerVerlag, Berlin, 1973), translated from German; original 1967.

${ }^{3}$ D. J. Mead, "A general theory of harmonic wave propagation in linear periodic systems with multiple coupling," J. Sound Vib. 27, 235-260 (1973).

${ }^{4} \mathrm{M}$. Ohlrich, "Wave propagation in periodic systems representing models of building structures: A theoretical and experimental study," Ph.D. thesis, Institute of Sound and Vibration Research, Southampton University, England, 1977.

${ }^{5} \mathrm{M}$. Ohlrich, "Harmonic vibration of a column structure with transverse beams," Proceeding of the Institute of Acoustics, Spring Meeting, Southampton, England, Paper 20.I2, 1979.

${ }^{6} \mathrm{H}$. L. Müller, "Biegewellen-Dämmung an symmetrischen und exzentrischen Sperrmassen" ("Attenuation of bending waves caused by symmetrical and eccentric blocking masses"), Dr.-Ing. Dissertation, Institut für Technische Akustik der Technischen Universität, Berlin, 1957.

${ }^{7}$ D. J. Mead and S. Markus, "Coupled flexural-longitudinal wave motion in a periodic beam," J. Sound Vib. 90, 1-24 (1983).

${ }^{8}$ M. Heckl, "Körperschallausbreitung auf Balken mit vielen Störstellen" ("Structure-borne sound propagation on beams with many discontinuities"), Acustica 81, 439-449 (1995).

${ }^{9}$ M. A. Heckl, "Acoustic properties of profiled cladding on buildings," Acust. Acta Acust. 87, 560-581 (2001).

${ }^{10}$ M. A. Heckl, "Coupled waves on a periodically supported Timoshenko beam," J. Sound Vib. 252, 849-882 (2002).

${ }^{11}$ A. K. Roy and R. Plunkett, "Wave attenuation in periodic structures," J. Sound Vib. 104, 395-410 (1986).

${ }^{12} \mathrm{D}$. J. Mead, "Wave propagation and natural modes in periodic systems: I. Mono-coupled systems," J. Sound Vib. 40, 1-18 (1975).

${ }^{13}$ D. J. Mead, "Wave propagation and natural modes in periodic systems: II. Multi-coupled systems, with and without damping," J. Sound Vib. 40, 19-39 (1975).

${ }^{14} \mathrm{~L}$. Friis and M. Ohlrich, "Coupled flexural-longitudinal wave motion in a finite periodic structure with asymmetrically arranged transverse beams," J. Acoust. Soc. Am. (in press).

${ }^{15}$ R. E. D. Bishop and D. C. Johnson, The Mechanics of Vibration (Cambridge University Press, Cambridge, 1960).

${ }^{16}$ G. L. Rodgers, Dynamics of Framed Structures (Wiley, New York, 1959).

${ }^{17}$ E. Rebillard and J. L. Guyader, "Vibrational behaviour of lattices of plates: Basic behaviour and hypersensitivity phenomena," J. Sound Vib. 205, 337-354 (1997).

${ }^{18} \mathrm{~S}$. Y. Lee and W. F. Yeen, "Free coupled longitudinal and flexural waves of a periodically supported beam," J. Sound Vib. 142, 203-211 (1990). 